\title{
Glaucoma, dementia, and the "precipice of care": transitions between states of medication adherence
}

\author{
Simon Read' \\ Heather Waterman' \\ James E Morgan ${ }^{2}$ \\ Robert A Harper 3,4 \\ Anne Fiona Spencer ${ }^{3}$ \\ Penelope Stanford ${ }^{5}$ \\ ISchool of Healthcare Sciences, \\ Cardiff University, Cardiff, UK; \\ ${ }^{2}$ School of Optometry and Vision \\ Sciences, Cardiff University, Cardiff, \\ UK; ${ }^{3}$ Manchester Royal Eye Hospital, \\ Manchester University NHS \\ Foundation Trust, Manchester, UK; \\ ${ }^{4}$ Division of Pharmacy and Optometry, \\ School of Health Sciences, University \\ of Manchester, Manchester Academic \\ Health Science Centre, Manchester, \\ UK; ${ }^{5}$ Division of Nursing, Midwifery \\ and Social Work, School of Health \\ Sciences, University of Manchester, \\ Manchester, UK
}

This article was published in the following Dove Press journal:
Patient Preference and Adherence

Purpose: "She wouldn't remember. Even when I go through, and she's decided to go to bed, I'll say I'll come and do her drops. If I didn't say that, they wouldn't be done." Dementia is widely considered as a key factor in whether patients take their medication as prescribed. However, few studies have examined the effect of dementia on medication management strategies for glaucoma including how patient and carer needs impact adherence and long-term prognosis. We report findings from a qualitative grounded theory study incorporating the views of patients, carers, and healthcare professionals.

Methods: Eighty-three semistructured interviews were conducted with 35 patients, 22 lay carers, and nine healthcare professionals across sites in Wales and Scotland. These explored understanding of eye drop regimens, barriers, and facilitators to drop administration, as well as attitudes toward glaucoma, dementia, and other comorbidities.

Results: Using Pound's synthesis of adherence behavior, we identified categories of active and passive acceptance of medicines, alongside modification or rejection of eye drop regimens. In relation to dementia, participants highlighted transitions between such categories, with a shift from active to passive acceptance commonly reported. This loss of self-medicating capability was referred to as the precipice of care, where entwinement of multiple conditions (eg, heart disease, glaucoma, and dementia) and sociocultural influences (eg, living alone) contributed to accelerated health declines. That said, numerous factors mitigated this, with a key role being the lay carer. Spouses and family members often acted as the monitor of eye drops for patients, seeking intervention when any behavioral changes influenced their administration.

Conclusion: Though dementia was associated with progression toward the precipice of care, factors such as communication with healthcare professionals appeared to affect patient adherence. Recommendations for healthcare practice include better recording of dementia diagnoses and integrating eye drops into preexisting routines.

Keywords: memory loss, compliance, eye drops, qualitative research, grounded theory

\section{Introduction}

The World Health Organization (WHO) 2003 report on adherence to long-term therapies emphasized concerns over patients failing to take medications as prescribed. ${ }^{1}$ It stated that the estimated worldwide adherence rate for chronic conditions was around $50 \%$, with this reducing clinical benefits and wasting medical resources. ${ }^{1}$ Research on nonadherence is ongoing, with numerous contributory variables identified, such as physical difficulties, psychosocial issues, side effects, beliefs about medication, organizational healthcare issues, and condition-specific factors. ${ }^{2}$ Therefore, adherence is a complex issue and, for each disease or person, various factors have different impacts. ${ }^{3}$
Correspondence: Simon Read

School of Healthcare Sciences, Cardiff University, Room 12.14, Eastgate House, 35-43 Newport Road, Cardiff CF24 OAB, UK

Tel +44292068 8930

Email readsm@cardiff.ac.uk 
Age-related conditions fit into the priorities set by the WHO, tending to be chronic and long term. ${ }^{1}$ Worldwide, the population aged 60 years or older is projected to rise by $56 \%$ from 901 million in 2015 to 1.4 billion in $2030 .{ }^{4}$ This trend is coupled with a rise in those older than 85 years, with the UK expecting this figure to double to 3.2 million by $2034 .{ }^{5}$ With this population growth comes the likelihood of increases in age-related conditions, prompting concerns of overstretched healthcare systems where prolonged self-medication is increasingly important. ${ }^{6}$ Though Banning noted poor adherence irrespective of age, for older people this can be particularly unpredictable with them often encountering complex regimens across multiple conditions, as well as adverse drug effects. It is estimated that while $45 \%$ of medicines prescribed in the UK are for older people, up to $50 \%$ of that population are nonadherent. ${ }^{8}$ Numerous studies have researched adherence behavior, with recent emphasis on the use of qualitative techniques to determine lay attitudes toward medication and the underlying causes for nonadherence. ${ }^{9}$ Many of these studies have helped to progress the field, offering functional models of active or passive adherence, resistance, and modification. ${ }^{10}$ However, few qualitative studies have examined this issue in relation to older people, with fewer still including people with dementia and how this pertains to the administration of medication. Arlt et al's $\mathrm{s}^{11}$ literature review aggregated much of the research on dementia and adherence, outlining the need to more fully examine patient, carer, and healthcare professional management strategies.

This article reports on qualitative, grounded theory research examining the effect of dementia on medication management for the degenerative eye condition, glaucoma. Internationally, glaucoma is one of the largest causes of permanent, severe visual impairment with an estimated 75 million people affected. ${ }^{12}$ In the UK context, glaucoma affects around 600,000 of those older than 65 years, although it is suggested that a further half of all cases may remain undiagnosed. ${ }^{12}$ The most common form of treatment for this condition is the instillation of daily eye drops that reduce the buildup of intraocular pressure responsible for damaging the optic nerve. ${ }^{13}$ Lifelong adherence to eye drops is a key concern for healthcare practitioners, given that the damage caused by glaucoma is irreversible. Similar to glaucoma, dementia is also a degenerative condition and affects around 47 million people worldwide, with this expected to be $\sim 75$ million by $2030 .{ }^{14}$ Dementia affects $\sim 815,000$ people older than 65 years in the UK, with this also expected to increase over time. ${ }^{15}$ The age-related nature of both conditions implies considerable overlap in the affected populations, with numerous authors highlighting the negative impact that dementia may have on adherence. ${ }^{16}$ No previous studies have explicitly examined this issue using qualitative methods that would identify common underlying behaviors and decision-making for patients, carers, and healthcare professionals. The findings have the potential to impact on national and international healthcare policies, particularly through the National Institute for Health and Care Excellence (NICE) guidelines on glaucoma diagnosis and management. ${ }^{17}$

\section{Methods}

The research study adopted a qualitative, grounded theory approach based on the requirement to study people's experiences and identify an underlying process, ${ }^{18}$ in this case, the management of eye drops for those affected by both dementia and glaucoma. Key features of grounded theory are as follows: 1) the simultaneous collection and analysis of data so that one informs the other; 2) targeted sampling to expand or verify the emerging theory; and 3 ) the constant comparison of new data with previously collected data to ensure the relevance of all to the theory. ${ }^{19}$ Numerous strategies were deployed to ensure the credibility of the study findings. These included consistency of methodological approach between and within research cohorts, meticulous record keeping ensuring the transparency of interpretation, and seeking out similarities and differences across accounts to enable the representation of different perspectives. ${ }^{41}$

\section{Data collection and sample}

Participants were recruited from glaucoma clinics in two countries, Wales and Scotland, between May 2016 and April 2017. Inclusion criteria for patients with dementia and glaucoma (patient cohort A) were as follows: aged 18 years or older, diagnosed with a form of glaucoma and dementia, able to provide informed consent according to the Mental Capacity Act 2005, and prescribed ocular hypotensive therapy. Patient records for those attending the glaucoma clinics were examined to confirm the diagnosis of dementia and identify potential participants in advance of appointments. A member of the clinical team informed attendees of the study and, if interested, introduced them to the research team for further information. Carers were eligible if aged 18 years or older and were providing lay care to someone with both glaucoma and dementia. In general, carers were recruited when attending the glaucoma clinic with the patient. The healthcare professionals were required to be aged 18 years or older and provide clinical care for those diagnosed with dementia and/or glaucoma.

We obtained an initial sample of 23 patients with a form of dementia and glaucoma (cohort A), 22 lay carers, and 
nine healthcare professionals. As the project progressed, an analytical theme on the relative importance of multiple conditions led to the study expanding to incorporate two additional patient cohorts. These comprised six patients experiencing just glaucoma (cohort B) and six patients who had glaucoma with another nondementia comorbidity (cohort C). From the total of 66 participants, 17 patients with dementia and glaucoma were interviewed twice in 6 months. Overall, this generated 83 semistructured interviews lasting between 30 and 60 minutes. The interview schedule explored the experiences of people with glaucoma and dementia, management and understanding of eye drop regimens, and any side effects, barriers, and facilitators to drop administration. These topics were deliberately kept flexible to allow the inclusion of unexpected themes in later interviews.

\section{Data analysis}

Thematic data analysis was carried out in line with the principles of grounded theory in which themes emerge as data are collected. ${ }^{20}$ NVivo 10 was used to organize and aggregate data within the developing framework. Analysis commenced by indexing the data and exploring topics considered important to broad medication adherence, before focusing specifically on glaucoma eye drops. The meaning of sentences or paragraphs was coded to construct a list of relevant categories. Each of these underwent constant comparative data analysis before being grouped into interrelated themes and concepts. During the analysis, memos were written between researchers to record developing ideas with regular research check-ins also established so as to reduce any potential for interpretative bias. ${ }^{41}$ These activities ultimately resulted in the expansion of the study to include the two further patient cohorts mentioned earlier. ${ }^{19}$ These interview transcripts were included in the wider data and were subjected to the same analytical approach. Theoretical sensitivity to other literatures was also important in providing insight into the emerging themes, with Pound et al's ${ }^{10}$ synthesis of qualitative studies offering an analytical filter to help understand the emergent data. Eventually, an overarching theory was generated relating all the concepts together, with key components including the influence of preexisting routines, attitudes toward medication, and the importance of carers in slowing deterioration.

\section{Ethics}

The study was granted ethical approval by the Wales 2 NHS Research Ethics Committee (Ref: 16/WA/0006) and approved by NHS Research and Development (R\&D) prior to the commencement of data collection. Written informed consent was obtained from all participants prior to the interviews taking place, with particular care taken for people with dementia. In these cases, capacity to consent was assessed according to the British Medical Association's (BMA) Mental Capacity Act Toolkit. This assessment included ensuring that participants understood and retained information relevant to the decision, used this to decide on participation, and were able to communicate their response. ${ }^{21}$ In addition, researchers used a form of "process consent" in which verbal agreement was sought throughout the interview schedule. ${ }^{22}$ Both the initial capacity assessment and ongoing process consent were also carried out during the second round of patient interviews. All quotations taken from the interview data were anonymized to prevent participant identification.

\section{Data set}

Tables 1-3 summarize the study participants from both research sites in Scotland and Wales. All of the patient participants were older than 70 years with just over half being

Table I Sample characteristics for patient cohorts A-C

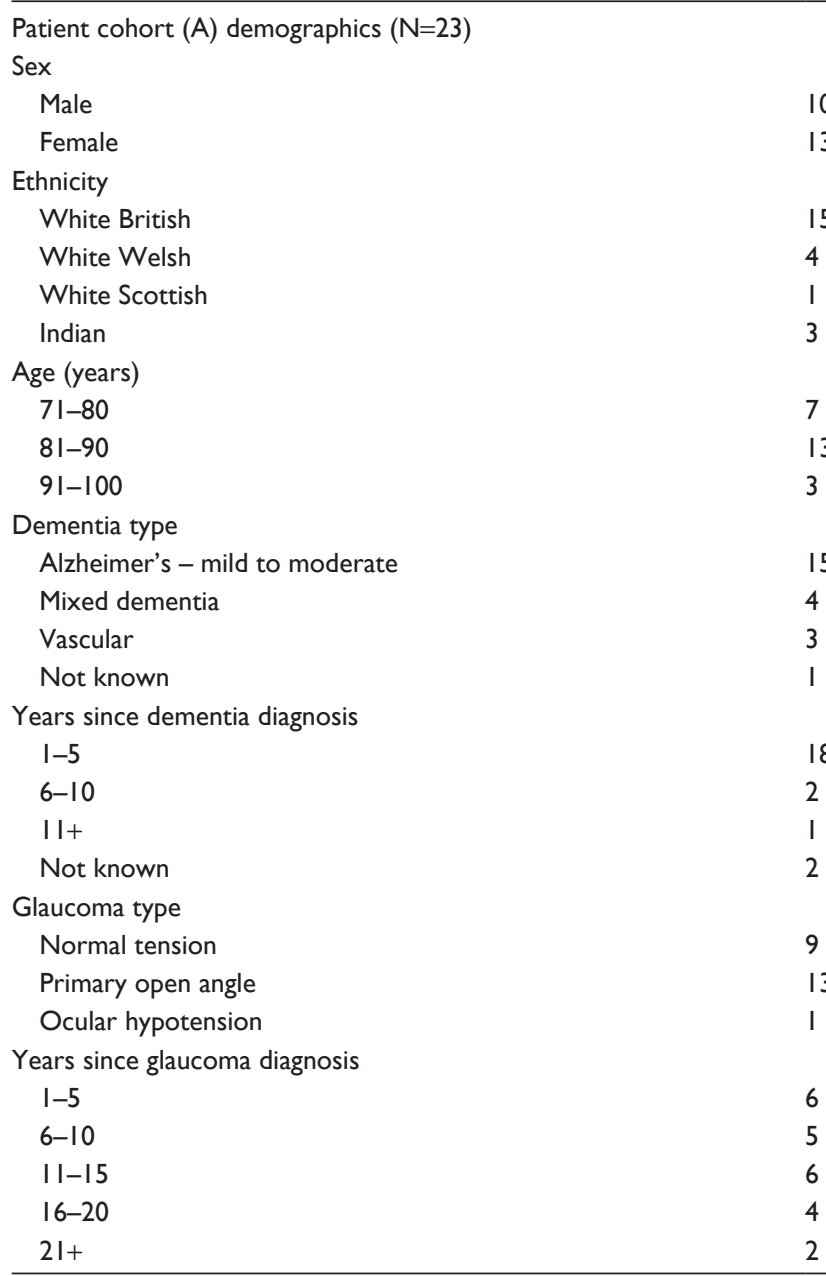

(Continued) 
Table I (Continued)

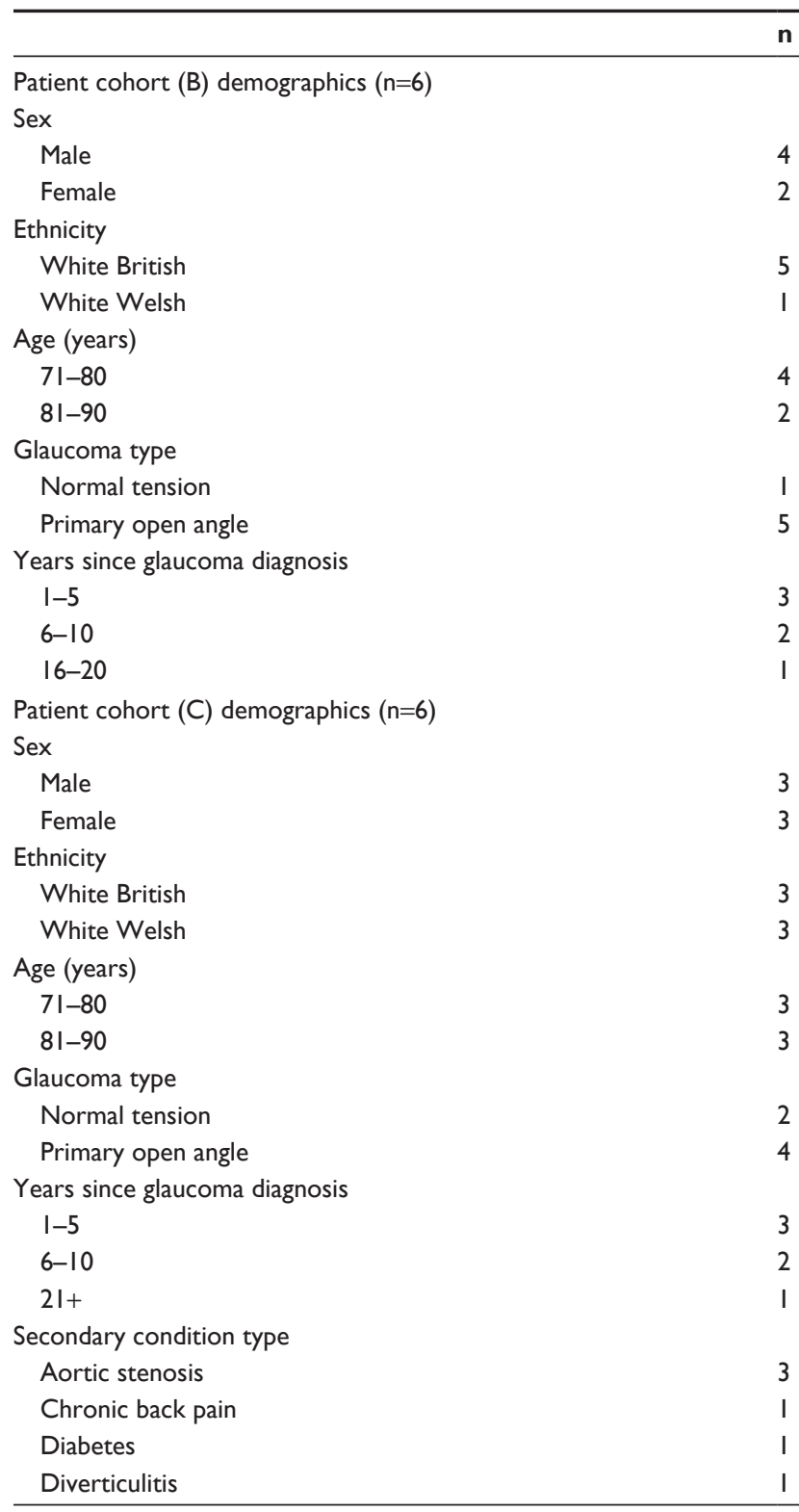

female. Two thirds of patients with dementia (cohort A) had been diagnosed with either mild or moderate Alzheimer's disease, with the remainder suffering from either vascular dementia or mixed dementia. Similarly, two thirds of the patients suffered from primary open angle glaucoma with the other third having normal tension glaucoma. This includes nondementia patient cohorts B (glaucoma only) and C (glaucoma and other comorbidities other than dementia) where comorbidities included aortic stenosis, diabetes, diverticulitis, and chronic back pain. The lay carer participants were generally family members with varying degrees of experience in caring for their proxies. Their ages ranged
Table 2 Sample characteristics for carer cohort

\begin{tabular}{|c|c|}
\hline Carer cohort demographics $(n=22)$ & $\mathbf{n}$ \\
\hline \multicolumn{2}{|l|}{ Sex } \\
\hline Male & 4 \\
\hline Female & 18 \\
\hline \multicolumn{2}{|l|}{ Ethnicity } \\
\hline White British & 18 \\
\hline White Welsh & I \\
\hline White English & I \\
\hline Indian & 2 \\
\hline \multicolumn{2}{|l|}{ Age (years) } \\
\hline $4 I-50$ & I \\
\hline $51-60$ & 7 \\
\hline $61-70$ & I \\
\hline $71-80$ & 8 \\
\hline $8 I-90$ & 4 \\
\hline Not known & I \\
\hline
\end{tabular}

between 50 and 90 years with the majority caring for one of the cohort A patients. Over three quarters of those in the lay caring role were female. The healthcare professionals comprised key members of the glaucoma clinic with roles including consultant, optometrist, nurse, and eye clinic liaison officer.

\section{Results}

Pound et al' ${ }^{10}$ synthesis of qualitative studies on medication adherence underpins the development of a model of behavior incorporating active or passive acceptance, rejection, and modification of prescriptions. These categories highlight the complexity of adherence management, with greater centrality given to lay understandings and attitudes. In line

Table 3 Sample characteristics for healthcare professional cohort

\begin{tabular}{ll}
\hline Healthcare professional cohort demographics $(\mathbf{n}=\mathbf{9})$ & $\mathbf{n}$ \\
\hline Sex & 2 \\
Male & 7 \\
Female & \\
Ethnicity & 8 \\
White British & I \\
Indian & \\
Age (years) & I \\
2I-30 & I \\
3 I-40 & 2 \\
$4 I-50$ & 5 \\
5 I-60 & \\
Job role & 2 \\
Consultant & 2 \\
Nurse & 3 \\
Optometrist & I \\
Eye clinic liaison officer & I \\
Support worker & \\
\hline
\end{tabular}


with the theoretical sensitivity associated with the grounded theory approach, Pound's model was useful in corroborating the study's emergent themes. Alignment was found between both studies through themes such as belief in the value of medication, minimizing intake, communication with healthcare professionals, and the influence of a carer on adherence. Thwaites ${ }^{23}$ has conceived of medication management as a continuum commencing from the initial prescription until the completion of the course. In chronic, degenerative conditions such as glaucoma and dementia, the course of medication is often lifelong, meaning that this continuum exists from diagnosis onward. However, as Insel et $\mathrm{al}^{24}$ notes, those experiencing dementia and decline in executive function may develop problems in formulating a plan to take their medicines, using assistive strategies and recalling that they took the medication. Our findings support this view, although a key focus was on transitions between states of adherence, how dementia affected this, and what mitigated or accelerated the transition.

\section{Categories of adherence}

Pound et $\mathrm{al}^{10}$ identified two primary categories of those who adhered to their medicine as prescribed, either passive accepters or active accepters. Interviews with each of our participant cohorts saw individuals falling into these categories, although there was also evidence of fluctuation between them.

\section{Active accepters}

Active accepters of medication have been described as "purposeful" adherers; a prerequisite of which is the perception that a prescription is necessary, effective, and safe. ${ }^{25}$ These factors promote a willingness to follow regimens, often also based on preexisting understandings of conditions and their consequences. In relation to glaucoma patients, similar ideas were evident:

Your eyesight is very precious. I would rather go deaf than lose my eyesight. So, oh no, I put the drops in. [WP15, Patient]

I've put dates on them, because they need to be used by a certain date ... your sight is precious, isn't it? So, I do what I'm told. [WPB02, Patient]

These extracts show respondents' acceptance of glaucoma medication. The quote from WP15 is indicative of how acceptance can be maintained through the early stages of memory loss. However, this theme was often more evident in the accounts of carers. One saw their mother maintaining fastidious adherence to medication over a period of several years before eventually declining:

She would really do it to the letter ... she used to say I have to take them the last thing of the day ... the consultant had told her that she should put them in and she should hold her fingers in her eyes for 60 seconds, and then once that was done, that was successful. [WC06, Carer]

This patient's dedication to adherence saw her following the consultant's instructions "to the letter". While the carer attributed this approach to the patient's attitudes toward their eyesight, she suggested that her deference to the health care professional also contributed. Indeed, another patient categorized as an active accepter offered a similar account when considering their own adherence:

I: Is it the fear of what would happen if you didn't take your medicine?

R: No, it's just the fact that they say, "Take this". [WP11, Patient]

Also, recurrent within the accounts of active accepters was the importance of preestablished routines, aligning with Insel et $\mathrm{al}^{24}$ and While et al. ${ }^{26}$ The extract below is from a carer outlining their mother-in-law's capability to instill eye drops after 5 years with mild Alzheimer's disease:

It's been a long term thing, it seems to be the one that's staying stable ... I think it's because in herself she's always had a routine, always kept a calendar. [WC10, Carer]

The carer felt that her mother-in-law's active acceptance and self-administration of the glaucoma drops were developed by the longevity of her routine, preestablished prior to the diagnosis of Alzheimer's disease. Further accounts of beneficial routines were evident where eye drops were integrated into wider medication regimens:

We're in a nice wee routine just now and I think it's fine that we keep that routine and we've gone over it again and again. [SC01, Carer]

So when I go to bed they're there on the landing. The last thing I see before I go into the bedroom is these rows of pills. [WP11, Patient]

Routines were often entwined with visual stimuli such as the strategic placement of eye drops and a visible record to aid memory, particularly for patients living alone. Carers also adopted these strategies upon realizing that memory loss may be affecting adherence. The placement of signs and calendars 
in familiar locations was reported by several carers, as well as the nonintrusive monitoring of medicine taking:

He does it himself. I've got to tell him, before we go to bed I say don't forget eye drops, in the fridge. [WC04, Carer]

When we come over, and that's once a week from Bris-

tol, I leave mum a little note on her bedside table to remind her to put the drops in that evening. [WC14, Carer]

Referring back to Thwaites's notion of a continuum, accounts of carers and patients outlined several phases of decline from active acceptance triggered by dementia. ${ }^{25}$ In both cases earlier, the carers expressed concern that reminders might lose their effectiveness or provoke greater dependence. WC14 went onto state that she found reminder notes discarded in a drawer, prompting concerns over forgetfulness. While these patients were actively self-administering, there was growing reliance on the carer to monitor and maintain independence.

\section{Passive accepters}

Pound et al's ${ }^{10}$ model suggested that passive accepters were either those who adhered indifferently toward their medication or those who relinquished control to others. The majority of passive accounts in this study fell into the latter category, with carers often recognizing that patients' medication management required intervention. This issue was particularly clear in a carer's account of her mother's declining fastidiousness:

She was very much in control of it until ... in the last two years ... her understanding of things became less, and then to remember to do things and what is this and why have you done that. [WC06, Carer]

This carer's mother had recently moved to a care home and was now entirely dependent on staff to instill her drops. The transition to sheltered housing or care home environments was evident in several cases and tended to coincide with medication not being remembered. The case below provided a snapshot of this as an emergent situation, with the carer beginning to realize that her mother's drops were being missed:

We have, with mum's permission, started to look at care homes ... not actually here in Wales but in England where we live. Because I am the only child, and we felt ... it would be better for it to be near us. [WC14, Carer, cited in interview ref: WP14]

A perceived change in cognition appeared to dictate the move to a care home with many elderly patients previously self-managing their medication. However, an awareness of deterioration tended to press the carer into investigating sheltered housing. For many, this decision was considerably stressful and often compromised long-established independence. Some preferred the patient to remain at home, instead arranging for care visits. There were, however, significant variations in the level of assistance provided by care staff; some patients were brought their eye drops to self-administer, while others required direct administration. Though generally determined by patient capability, the care home or visiting staff also influenced the mode of administration:

I'm hoping that maybe the carers can ... they're not allowed to administer the eye drops but I'm hoping I can try and get through to them that even if they could stand over her while she takes them. [WC01, Carer]

In this case, visiting carers were unqualified to administer eye drops and could not mitigate the risk of nonadherence. Though verbal reminders were generally provided, these were not considered effective. However, in other cases, the transition to passive adherence was smoother as drops were brought to the patient and instilled independently. Moving to a care home or integration of visiting carers was more common in the absence of a live-in carer, such as spouse and a family member. Son or daughter carers were sometimes unable to visit the patient every day, which when coupled with doubts about adherence capability, led to more complex arrangements. However, in cases where elderly patients lived with their spouse, the management of medications could become part of their caring role:

She wouldn't remember. Even when I go through, and she's decided to go to bed, I'll say I'll come and do her drops. If I didn't say that, they wouldn't be done. [WC03, Carer]

This extract suggests that memory loss prevented the carer's wife from taking her drops. In addition, her transition into passive adherence was related to her inability to self-administer the drops accurately, often missing her eye. Indeed, the shift to passive adherence often appeared to be less related to forgetfulness and more to preexisting dependence between patient and carer:

I've got no idea of whether I'd get my eye. I just hold them back and she drops them in. [WP06, Patient]

The way I manage is my wife says come here, you've got to put your head back and she puts the drops in. [WP07, Patient]

These extracts were taken from passively adherent patients reliant on their spouses for eye drop administration. 
While other accounts demonstrated cognitive and physical decline were heavily influential, these were not the primary trigger in these cases. Instead, it was reported that patients were dependent on their spouses for direct administration from diagnosis onward, even though physically capable. This suggested that dependence on a carer may be influenced by preexisting attitudes toward medicine taking and dynamics in the patient-carer relationship.

\section{Categories of nonadherence}

Both categories of medication acceptance reported eye drops being instilled successfully. Nonadherence was also reported in some cases, particularly in the transition between activity and passivity. The NICE 2009 guidelines on adherence categorize this as unintentional nonadherence, driven by forgetfulness. ${ }^{27}$ Within the same guidelines, intentional nonadherence is characterized by the "beliefs and preferences that influence the person's perceptions of the treatment". ${ }^{27}$ Pound et $\mathrm{a} \mathrm{l}^{10}$ provided insight into these lay beliefs, focusing on the rejection and modification of regimens. The former category is characterized by skepticism over the medication, leading to conscious and purposeful nonadherence; the latter comprised those who actively adapt their intake. ${ }^{10}$

\section{Rejection}

Accounts of rejection of medication were relatively uncommon for those with dementia. The majority of participants expressed acceptance of their eye drops, with this being compromised by memory loss. Nevertheless, thematic analysis revealed several attitudinal factors influencing the adoption of strategies to manage eye drops. Taking the condition of glaucoma seriously was a key prerequisite to active acceptance, yet some participants appeared to deprioritize it:

$\mathrm{R}$ : She does it when she thinks about it. When you remember you do it, don't you?

R2: I'm a bit naughty ...

$\mathrm{R}$ : Because it isn't giving her any trouble, she isn't bothered with it, you know? [WC02, Carer (R) and WP01, Patient (R2)]

This elderly couple self-reported inconsistent adherence to eye drops, corroborated by their daughter referencing "two carrier bags" full of unused medication (WC01, Carer). While forgetfulness was mentioned, it was also apparent that glaucoma was not provoking any noticeable visual problems for the patient, prompting a more ambivalent response to treatment. Previous studies have highlighted the asymptomatic nature of glaucoma contributing to nonadherence, with longterm impacts initially unclear. ${ }^{28}$ This was further heightened by the presence of other comorbidities including dementia and arthritis, each of which appeared to be considered more important than glaucoma:

Well, in fact, I don't give her eyes a thought actually because they aren't that important. No, it's more like other things take priority over them at the moment. [WC02, Carer]

Glaucoma's deprioritization relative to other conditions was apparent across all patient cohorts, both with and without dementia. For those with symptoms of memory loss, these were often regarded as the primary concern. That said, for the majority of this cohort, carers helped to reestablish eye drops as important by encouraging and reminding their proxy. The extract above suggests that if carer and patient deprioritize the condition, there is a greater likelihood of nonadherence. In contrast, another patient with dementia believed that he could remember eye drops but remained apathetic:

I: Would you have problems remembering to take your eye drops if your wife wasn't here to help you?

R: Not really. I probably wouldn't bother. [WP21, Patient]

The patient reiterated his ambivalence was responsible for historic nonadherence to eye drops throughout the interview. Here, the patient lived with his wife who now instilled the drops, commencing a period of passive acceptance. Nevertheless, the patient's attitude to glaucoma was in stark contrast to his other conditions. Indeed, many of those with a condition other than dementia also noted their prioritization of these over glaucoma:

You can pop off quickly with the heart and you don't pop off quickly with glaucoma. [WPC01, Patient]

I know part of my colon is affected and I'm going to have part of that removed ... but that affects me, the glaucoma and the cataracts don't really affect me. [WPC04, Patient]

I: Which of the conditions would you say concerns you the most?

R: I suppose the cardiac problem ... I don't think of my eyes at all. [WPC06, Patient]

For some participants, glaucoma appeared to be considered less serious than other conditions, potentially influencing attitudes toward eye drops. Nevertheless, the majority of participants did not reject the medication outright. The only instance in which eye drops were abandoned altogether came from a patient with chronic back pain:

One stage playing golf my eyes watered excessively ... for some reason I stopped the prescribed drops ... I got told 
off about that because apparently my eye pressure went up.

[WPC05, Patient]

Interestingly, unlike many accounts of acceptance where carers and patients saw association between glaucoma and blindness, this patient rejected the medication without understanding the consequences. This implies inadequate patient education at diagnosis which, when experiencing side effects, triggered a period of nonadherence. The interviewee went onto outline that positive interactions with healthcare professionals altered this behavior, highlighting the influence of clear, person-centered communication within the clinic.

\section{Modification}

Pound et al's category of modifiers suggested active engagement from patients, with medicine being taken but not necessarily as prescribed. The motivations for patients to modify their regimen may be preexisting suspicion toward it, side effects, or minimizing the impact that it has on their lives. ${ }^{10}$ Accounts of modification behavior were less pervasive within the interviews than those of active or passive acceptance. Nevertheless, some patients sought to shift their medication patterns:

The lady that I saw when I saw you before, I said that if I go out for a pint I'll put them in before I go. She said that's a good idea as when you come back you can forget it. [WPC06, Patient]

This patient modified his prescription on evenings where he went out socializing. This was agreed with a member of the glaucoma clinic suggesting that cooperation between patient and health care professional could optimize medication intake. Many healthcare professionals stated that they would rather have patients modify their routines if it encouraged eye drop use:

I've got some patients who just can't remember to do things in the morning ... or vice versa ... if you were just to say well do it all together rather than being fixed ... I think that would make life a lot easier. [WH02, Optometrist]

This person-centered flexibility to regimens demonstrates how healthcare professionals, carers, and patients can collaborate to identify the most appropriate, personalized routines. ${ }^{27}$ In terms of patients with dementia, flexibility could also facilitate better intake of eye drops. The value of routines for both active and passive accepters of the medication has already been outlined, and as the following example shows, disruption or deviation can result in poorer levels of adherence:
When he was putting them in at night time, he was used to

it. But now because of the change in the time, he's more forgetful. [WC12, Carer]

This carer reported that a healthcare professional suggested moving her husband's eye drops from the evening to the morning. This was intended to maximize the effectiveness of the drops but ultimately resulted in less adherence and an additional burden on the carer to monitor intake. The participants believed that the healthcare professional was unaware of the husband's diagnosis of dementia yet passively accepted their instructions. Knowledge of the patient's comorbidities may have prevented advice that could compromise rather than benefit medication adherence. Open, person-centered discussion between carers, patients, and health care staff may help to ensure that all parties agree the best management strategy.

\section{Discussion: adherence transitions and the precipice of care}

Thwaites's ${ }^{23}$ continuum of care presents adherence as a linear path from prescription to completion of the course. While Pound et al's ${ }^{10}$ categories of adherence and nonadherence offer a functioning snapshot of a patient's status, the factors influencing transitions between them are less transparent. This discussion centers on how dementia affects these transitions, while also examining factors relevant to those with and without memory loss. The most common shift in reported adherence behavior was from a state of active to passive acceptance. Carers who had witnessed a decline in the patient's ability to self-manage medication described the progression as being from independence to partial and then complete dependence. Even so, numerous carers referenced long spells of fastidious adherence that ran counter to the predicted pathway, with the patient sustaining self-management many years postdiagnosis. Variables such as type and severity of dementia naturally contribute to the experienced decline, but the points outlined below were reported as having potential to slow progress toward a precipice of care.

\section{Belief in value of medication and healthcare system}

Belief in the value of medication and the wider healthcare system is associated with active management of medication. ${ }^{10,25}$ The majority of participants regarded medication as important, and in relation to glaucoma care, this belief was generally predicated on understanding the condition. Many adherent patients expressed anxiety over the potential for glaucoma to develop into blindness and 
how this explained their fastidious use of eye drops. This aligns with findings from various eye drop adherence studies where those who saw the necessity of taking drops were more adherent. ${ }^{29,30}$ The counterpoint to this was believing that eye drops were unnecessary, commonly linked to a lack of patient's awareness of the condition. ${ }^{31}$ Only one participant expressed this view, reporting a spell of nonadherence after encountering watery eyes. In this case, treatment was resumed following an appointment in the glaucoma clinic where healthcare professionals reasserted the medication's importance. Interestingly, this patient did not have dementia, suggesting that educational interventions may be useful for all patients receiving a glaucoma diagnosis. For people with dementia and glaucoma, a belief in the value of eye drops did not necessarily prevent the transition from active to passive acceptance or nonadherence. Nevertheless, continued eye drop use was dependent on the perception of glaucoma as a serious condition.

\section{Relative importance of conditions and comorbidities}

Older people often experience multiple comorbidities, which can be problematic both for medication adherence and the care burden. ${ }^{32,33}$ Many participants had conditions other than glaucoma and dementia, with arthritis and cardiac issues being particularly common. For some of these participants, adherence to glaucoma drops was deprioritized relative to other conditions. Numerous authors have indicated how adherence to medication can be guided by symptomatic experience. ${ }^{25,34}$ Glaucoma care does not fit neatly within this on the basis that is generally asymptomatic and characterized by gradual visual decline. ${ }^{28}$ This model of symptomatic progression appeared to impact on the importance attributed to eye drops compared with other medications, more so if the care burden was particularly heavy. A greater understanding and awareness of glaucoma was associated with a belief in the value of medication to prevent its progression on the part of patients and carers. Healthcare professionals looking to improve adherence should be looking to identify this set of patients, be aware of their healthcare requirements, and attempt to integrate the drops into their broader, preexisting medication suites.

\section{Preestablished routines}

The perceived value of routines in dementia care has been widely documented in the healthcare community. ${ }^{35,36}$ Supporting data have documented participant's engagement with long-established routines after a dementia diagnosis.
Many of those diagnosed with glaucoma before dementia maintained their eye drop regimen, apparently due to it being embedded in their daily activities. Carers often stated that patients' prediagnosis life had involved the daily use of diaries and calendars, which they felt prolonged the phase of active acceptance. The importance of this also emerged when patients sought to modify their regimen. For people with dementia, this often involved integrating drop administration with other established routines. The use of time-based blister packs helped many with dementia orientate themselves to their pills, often alongside other memory aids. Eye drops, however, do not fit within such packs leaving them at risk of being overlooked. Future studies should consider integrating visual aids such as stickers onto blister packs, representing the requirement to take eye drops for those with memory-driven adherence issues. This approach would be dependent on healthcare professionals offering person-centered flexibility and recognizing that general prescription advice may not be applicable for all patients.

\section{Presence of carer}

Lay carers of people with dementia are widely acknowledged for their role in medication management. ${ }^{37,38}$ The study data reflected this with numerous instances where spouses or other family members assumed control of medication management once passive acceptance was reached. However, the carers were also shown to assist with independent self-management through monitoring and reminders. Carers were also helpful in identifying subtle shifts in behavior that may affect the transition between adherence categories. This tacit knowledge extended to the patient's preferences and routines, helping to inform modifications beneficial to intake. Even so, the role of carer is associated with a considerable burden that often goes unacknowledged by healthcare professionals. ${ }^{39}$ Given the importance of carers in identifying and preventing transitions between states of adherence, providing adequate support is essential. Several carer participants engaged with organizations such as Alzheimer's Society, Age UK, or similar services suggested by their general practitioner. Activities included counseling, communication strategies, and access to a network of others with dementia. These were widely reported as helpful, suggesting that healthcare professionals could provide further information on such services if aware of a dementia diagnosis. The importance of the carer in disclosing the diagnosis of dementia was raised by numerous healthcare professionals. Glaucoma clinics are extremely busy with little time to study patient records and identify dementia diagnoses, should they even be routinely recorded. 
The identification and awareness of dementia are among the first steps in healthcare professionals providing appropriate strategies for medication and other aspects of daily life. ${ }^{11,26}$

It is worth noting that the value of carers to medication management is most clear in cases where they are absent. Progression toward a precipice of care is more likely where patients are living alone with minimal input from carers in spite of varying levels of capability beyond dementia. The study showed several participants with dementia acting as carer to their spouse based on the latter's own health issues. Where both members of an elderly couple are unable to provide adequate care for one another, there is a much greater chance of inexorable decline. For glaucoma, these cases could easily lie undetected where appointments are often separated by 6-12 months.

\section{Conclusion}

The present study explored the adherence behaviors of those experiencing both glaucoma and dementia and the effect that dementia may have on transitions between states of adherence. While the study was limited to patients with mild dementia able to provide their own informed consent, the findings offer valuable insights into practical adherence strategies during this phase of the condition, as well as for nondementia patients. These qualitative findings should be corroborated in the wider literature given the relatively small sample being investigated. Nevertheless, this study's contribution to the field has shown that dementia is associated with shifts in adherence with many active acceptant participants moving to passively adherent or nonadherent states postdiagnosis.

Numerous factors have been outlined that may slow adherence transitions. The embedding of routines, engagement with support organizations, and the presence of a carer, alongside attitudinal elements such as believing in the value of medication and taking the progressive nature of the condition seriously, appear to have positive mitigating effects. Additionally, a positive relationship between patient, carer, and health care professional was associated with better medication management, allowing person-centered modifications to prescribed regimens based on lifestyle patterns and preferences. This negotiated alliance may be most appropriate to people with dementia, with the collective care team identifying the optimal method to ensure that medication is prescribed according to individual needs rather than population-wide guidance. ${ }^{10,40}$ However, this approach is dependent on healthcare professionals' knowledge of the dementia diagnosis, something often overlooked in the glaucoma clinic. Nevertheless, the value of offering improved education for all glaucoma patients, regardless of dementia diagnosis, should be emphasized. The study data demonstrated the potential for those with and without dementia to benefit from enhanced learning on the natural progression and consequences of glaucoma.

In relation to eye drops, specific issues emerged that arguably contributed to patients becoming nonadherent, such as eye drops not easily fitting into aids such as time-based blister packs. The use of stickers as visual reminders on such packs, or the provision of preservative-free, single dose drops where appropriate, was seen as a potential means to integrate eye drops into broader medication suites. Other cases showed that the manual dexterity and strength required for eye drop instillation was lacking in patients with arthritis. Addressing these issues is predicated on the knowledge of their existence, emphasizing once more the importance of a carer in their identification and the value of clear communication between carers, patients, and healthcare professionals.

\section{Acknowledgment}

This research study was funded by the International Glaucoma Association in conjunction with the Royal College of Nursing.

\section{Disclosure}

The authors report no conflicts of interest in this work.

\section{References}

1. World Health Organisation [webpage on the Internet]. Adherence to Long-Term Therapies: Evidence for Action; 2003. Available from: http://www.who.int/chp/knowledge/publications/adherence_report/en. Accessed March 1, 2018.

2. Osterberg L, Blaschke T. Adherence to medication. $N$ Engl J Med. 2005;353:487-497.

3. Vermeire E, Hearnshaw H, Van Royen P, Denekens J. Patient adherence to treatment: three decades of research: a comprehensive review. J Clin Pharm Ther. 2001;26(5):331-342.

4. United Nations, Department of Economic and Social Affairs, Population Division. World Population Ageing 2015. New York: United Nations; 2015.

5. Office of National Statistics. National Population Projections: 2014Based Statistical Bulletin. London: United Nations; 2015.

6. Oliver D, Foot C, Humphries R. Making Our Health and Care Systems Fit for an Ageing Population. London: The King's Fund; 2014.

7. Banning M. Older people and adherence with medication: a review of the literature. Int J Nurs Stud. 2008;45(10):1550-1561.

8. SCIE. SCIE Research Briefing 15: Helping Older People to Take Prescribed Medication in Their Own Home: What Works? London: SCIE; 2005.

9. Phatak H, Thomas J. Relationships between beliefs about medicines and nonadherence to prescribed chronic medications. Ann Pharmacother. 2006;40:153-159.

10. Pound P, Britten N, Morgan M, et al. Resisting medicines: a synthesis of qualitative studies of medicine taking. Soc Sci Med. 2005;61(1): $133-155$. 
11. Arlt S, Lindner R, Rosler A, et al. Adherence to medication in patients with dementia. Drugs Aging. 2008;25(12):1033-1047.

12. International Glaucoma Association [webpage on the Internet]. Prevalence: How Common Is Glaucoma? 2018. Available from: http:// www.glaucoma-association.com/about-glaucoma/what-is-glaucoma/ prevelence. Accessed March 1, 2018.

13. International Glaucoma Association [webpage on the Internet]. Treatments; 2018. Available from: https://www.glaucoma-association.com/ about-glaucoma/treatments. Accessed March 1, 2018.

14. Prince M, Wimo A, Guerchet M, Ali G, Wu Y, Prina M. World Alzheimer Report 2015-The Global Impact of Dementia: An Analysis of Prevalence, Incidence, Costs and Trends. London: Alzheimer's Disease International; 2015.

15. Alzheimer's Society. Dementia UK: Update. London: Alzheimer's Society; 2014.

16. Jain S, Ahmad A. Senile dementia and glaucoma: evidence for a common link. J Ophthalmic Vis Res. 2015;10(2):178-183.

17. National Institute for Health and Care Excellence. Glaucoma: Diagnosis and Management. London: National Institute for Health and Care Excellence; 2017.

18. Lingard L, Albert M, Levinson W. Grounded theory, mixed methods, and action research. Br Med J. 2008;337:567.

19. Glaser B, Strauss A. The Discovery of Grounded Theory: Strategies for Qualitative Research. Chicago: Aldine; 1967.

20. Glaser B. Gerund Grounded Theory: The Basic Social Process Dissertation. Mill Valley: Sociology Press; 1996.

21. British Medical Association [webpage on the Internet]. Assessing Mental Capacity; 2016. Available from: https://www.bma.org.uk/ advice/employment/ethics/mental-capacity/assessing-mental-capacity. Accessed March 1, 2018.

22. Dewing J. Participatory research: a method for process consent with persons who have dementia. Dementia. 2007;6(1):11-25.

23. Thwaites J. Practical aspects of drug treatment in elderly patients with mobility problems. Drugs Aging. 1999;14(2):105-114.

24. Insel K, Morrow D, Brewer B, Figueredo A. Executive function, working memory, and medication adherence among older adults. J Gerontol. 2006;61B(2):102-107.

25. Johnson M, Williams M, Marshall E. Adherent and nonadherent medication-taking in elderly hypertensive patients. Clin Nurs Res. 1999;8(4): 318-325.

26. While C, Fleur D, Beanland C, Koch S. Medication management: the perspectives of people with dementia and family carers. Dementia. 2012;12(6):734-750.
27. National Institute for Health and Care Excellence. Medicines Adherence: Involving Patients in Decisions about Prescribed Medicines and Supporting Adherence. London: National Institute for Health and Care Excellence; 2009.

28. Schwartz G, Quigley H. Adherence and persistence with glaucoma therapy. Surv Ophthalmol. 2008;53(1):S57-S67.

29. Tsai J, McClure C, Ramos S, Schlundt D, Pichert J. Compliance barriers in glaucoma: a systematic classification. J Glaucoma. 2003;12(5): 393-398.

30. Stryker J, Beck A, Primo S, et al. An exploratory study of factors influencing glaucoma treatment adherence. J Glaucoma. 2010;19(1): 66-72

31. Lacey J, Cate H, Broadway D. Barriers to adherence with glaucoma medications: a qualitative research study. Eye. 2009;23(4):924-932.

32. Tadd W, Hillman A, Calnan M, Calnan S, Read S, Bayer A. From right place - wrong person, to right place - right person: dignified care for older people. J Health Serv Res Policy. 2012;17(2):30-36.

33. Calnan M, Tadd W, Calnan S, Hillman A, Read S, Bayer A. 'I often worry about the older person being in that system': exploring the key influences on the provision of dignified care for older people in acute hospitals. Ageing Soc. 2013;33(3):465-485.

34. Angermeyer M, Loffler W, Muller P, Schulze B, Priebe S. Patients' and relatives' assessment of clozapine treatment. Psychol Med. 2001;31: 509-517.

35. Askham J, Briggs K, Norman I, Redfern S. Care at home for people with dementia: as in a total institution? Ageing Soc. 2007;27:3-24.

36. Rabins P, Lyketsos C, Steele C. Practical Dementia Care. Oxford: Oxford University Press; 2016.

37. Erlen J, Lingler J, Sereika S, Tamres L, Happ M, Tang F. Characterizing caregiver-medicated medication management in patients with memory loss. J Gerontol Nurs. 2013;39:30-39.

38. Gillespie R, Mullan J, Harrison L. Managing medications: the role of informal caregivers of older adults and people living with dementia A review of the literature. J Clin Nurs. 2014;23(23-24):3296-3308.

39. Svendsboe E, Terum T, Testad I, et al. Caregiver burden in family carers of people with dementia with Lewy bodies and Alzheimer's disease. Int J Geriatr Psychiatry. 2016;31:1075-1083.

40. Waterman H, Brunton L, Fenerty C, Mottershead J, Richardson C, Spencer F. Adherence to ocular hypotensive therapy: patient health education needs and views on group education. Patient Prefer Adherence. 2013;7:55-63.

41. Noble H, Smith J. Issues of validity and reliability in qualitative research. Br Med J. 2015;18(2):34-35.
Patient Preference and Adherence

\section{Publish your work in this journal}

Patient Preference and Adherence is an international, peer-reviewed, open access journal that focuses on the growing importance of patient preference and adherence throughout the therapeutic continuum. Patient satisfaction, acceptability, quality of life, compliance, persistence and their role in developing new therapeutic modalities and compounds to optimize

\section{Dovepress}

clinical outcomes for existing disease states are major areas of interest for the journal. This journal has been accepted for indexing on PubMed Central. The manuscript management system is completely online and includes a very quick and fair peer-review system, which is all easy to use. Visit http://www. dovepress.com/testimonials.php to read real quotes from published authors. 